\title{
Efficacy of rhizobacteria for growth promotion in sorghum under greenhouse conditions and selected modes of action studies
}

\author{
A. IDRIS ${ }^{1 *}$, N. LABUSCHAGNE ${ }^{2}$ AND L. KORSTEN ${ }^{2}$ \\ ${ }^{1}$ Department of Microbiology and Plant Pathology, University of Pretoria, 0002 Pretoria, South Africa \\ ${ }^{2}$ Department of Biology, Alemaya University, Diredawa 138, Ethiopia
}

(Revised MS received 11 June 2008; First published online 13 November 2008)

\begin{abstract}
SUMMARY
The screening of rhizobacteria for growth promotion of sorghum (Sorghum bicolour (L.) Moench) was conducted under greenhouse conditions for a total of 78 bacteria isolated from the rhizosphere of sorghum in Ethiopia, and 86 isolates from the rhizosphere and rhizoplane of grasses in South Africa. Three isolates from Ethiopia, all identified as Bacillus cereus, enhanced growth promotion by resulting in statistically significant increases in at least five parameters. Of these, B. cereus (KBE7-8) resulted in significant increase in shoot and root biomass. Among effective isolates from South Africa, B. cereus (NAS4-3) and Stenotrophomonas maltophilia (KBS9-B) showed significant increases in all the parameters measured. The isolates which resulted in significant growth promotion colonized the roots effectively with a count up to $\geqslant 10^{8} \mathrm{cfu} / \mathrm{g}$. In the study conducted to elucidate the possible modes of action by these effective isolates, indole 3-acetic acid-like substances were detected in culture filtrates of the isolates ranging from $4 \cdot 2 \mu \mathrm{g} / \mathrm{ml}$ by Serratia marcescens (KBS9-R) to $22 \cdot 8 \mu \mathrm{g} / \mathrm{ml}$ by B. cereus (KBS5-H) in the presence of $2 \mathrm{mg}$ tryptophan/g nutrient broth solution. Higher rates of solubilization of tricalcium phosphate on Pikovskaya agar medium were shown by Chryseomonas luteola (KBS5-F), S. marcescens (KBS6-H) and B. cereus (KBE9-1). There is very limited knowledge of the use of rhizobacteria in agriculture in Ethiopia and South Africa. The current study therefore generates valuable information towards application of plant growth promoting rhizobacteria as alternatives to chemical fertilizers.
\end{abstract}

\section{INTRODUCTION}

Sorghum (Sorghum bicolour (L.) Moench) ranks fifth among the World's major cereals following wheat, maize, rice and barley (House 1995; FAO 1999). This dry land summer cereal is a staple crop in arid and semi-arid areas in Ethiopia. It is also a staple food for more than 500 million people in more than 30 countries, although maize has often replaced its use. In Ethiopia, where traditional agriculture predominates, the average yield is very low, ranging between 200 and $1500 \mathrm{~kg} / \mathrm{ha}$ compared to developed countries such as the USA where commercial yields were 3775$4400 \mathrm{~kg} / \mathrm{ha}$ in the 1980s (House 1995). According to a recent report (FAOSTAT 2005), sorghum yields in

* To whom all correspondence should be addressed. Email: ahmed.idris@fabi.up.ac.za
2005 were $9800 \mathrm{~kg} / \mathrm{ha}$ in the USA but only $1800 \mathrm{~kg} / \mathrm{ha}$ in Ethiopia. Diseases caused by fungal pathogens (Huluka \& Esele 1992; Davis \& Bockus 2001) and unavailability of essential nutrients such as phosphorous and iron (Rodriguez \& Fraga 1999; Igual et al. 2001) are among some of the major causes of the low sorghum yield in developing countries.

Beneficial bacteria can be a significant component in the management of the soil environment so as to achieve attainable crop yield (Cook 2002). These beneficial bacteria live in the rhizosphere, the region around the root, which is rich in nutrients due to the exudation of 0.40 of plant nutrients from the roots (Nelson 2004). By benefiting from the nutrients secreted by plant roots within the rhizosphere, the bacteria influence the plants in a direct or indirect way. One influence may be stimulation of plant growth (Bloemberg \& Lugtenberg 2001). Bacteria 
inhabiting the rhizosphere and positively influencing plant growth are referred to as plant growth promoting rhizobacteria (PGPR) (Kloepper et al. 1986).

Significant yield increases have been achieved in crops such as maize, rice, potato, wheat and canola after inoculation with PGPR (Khalid et al. 1997; Zahir et al. 1998; Bertrand et al. 2001; Thakuria et al. 2004) which resulted in increased interest in PGPRs (Asghar et al. 2004; Thakuria et al. 2004). There are several hypotheses about the mechanisms by which rhizobacteria enhance growth. One direct mechanism is production of the auxin indoleacetic acid (IAA) (Patten \& Glick 1996, 2002). Another direct mechanism may be increased availability of nutrients in the rhizosphere by means of solubilization of unavailable forms of nutrients and/or production of siderophores (Glick 1995; Rodriguez \& Fraga 1999). Free living diazotrophic bacteria such as Azosprillum also are involved in promoting the growth of many tropical grasses, by fixing nitrogen asymbiotically and transferring it to the plant (Saubidet et al. 2002).

Although there is a growing interest in replacing chemical fertilizers and pesticides with bacterial inoculants (Mayak et al. 2001), there have been few laboratory or field studies of the potential role of PGPRs as plant growth promoting agents in Ethiopia and South Africa. The objectives of the present study are therefore to isolate bacteria from the rhizosphere of sorghum and other grasses in Ethiopia and South Africa, evaluate them for growth promotion of sorghum under greenhouse conditions and investigate selected modes of action used by the bacteria. It is anticipated that the study will provide important information toward application of PGPRs as inoculants in agriculture.

\section{MATERIALS AND METHODS}

\section{Soil sample collection and isolation of bacteria}

Soil samples were collected from the rhizosphere of sorghum in two fields in Ethiopia, in the Meeson and Jijiga areas in the Eastern part of the country. The soils have been frequently cropped with sorghum and differ in their texture. Sorghum plants were uprooted and all the soil adhering to the roots and which represent rhizosphere soil were shaken from the roots and collected in plastic bags. The soil samples were then transported to the microbiology laboratory of the Department of Biology, Alemaya University, Ethiopia for immediate processing.

To isolate bacteria, each soil sample was mixed and one gram was transferred to $9 \mathrm{ml}$ quarter strength sterile Ringer's (Merck, Halfway House, South Africa) solution and was serially diluted. Diluted suspensions were spread-plated on King's B medium (King et al. 1954) and Nutrient agar medium (Biolab, Wadeville, South Africa) in triplicate and were incubated for $24 \mathrm{~h}$ at $28^{\circ} \mathrm{C}$. Representative colonies were randomly selected from the countable plates and re-streaked onto new plates of the same media to obtain pure colonies. A total of 142 isolates obtained in this manner were maintained on agar slants and transported to the Plant Pathology Laboratory at the Department of Microbiology and Plant Pathology, University of Pretoria, South Africa. Because many isolates were morphologically indistinguishable in culture, preliminary characterization procedures including Gregersen's KOH (Gregersen 1978), cytochrome oxidase (Kovacs 1956), oxidation fermentation (Hugh \& Leifson 1953), catalase and motility tests were conducted. Based on these preliminary characterizations, 78 isolates were chosen from the original 142 isolates. For short term use, pure cultures of these isolates were stored at $-70{ }^{\circ} \mathrm{C}$ in nutrient broth supplemented with $150 \mathrm{mg} / \mathrm{g}$ glycerol. Replicate stocks of the cultures were lyophilized and stored for long term use.

Samples also were collected from ten selected sites of the virgin soil from the rhizosphere of grasses in the Nylsvlei Nature Reserve in South Africa. The 4000 ha reserve lies east of the Waterberg Mountains between Nylstroom and Naboomspruit possessing a unique biodiversity of plant and animal communities. Soil samples were collected as described before from the rhizosphere of seven grass species viz. Aristida canescens subsp. canescens (perennial), Cyprus esculentus L. (perennial), Eragrostis biflora (annual), Eragrostis sp. (annual), Sporobolus fimbriatus (perennial), Stipaggrotis zeyheri subsp. zericans (perennial), Themeda triandra (perennial) of the typical bushveld savannah surrounding the grassveld flood plain. The samples were transported to the Laboratory at the Department of Microbiology and Plant Pathology, University of Pretoria, South Africa. Each sample of rhizosphere soil was sieved to remove plant debris.

To isolate bacteria from samples obtained from the rhizosphere of grasses, the soil samples were baited with sorghum seeds in the greenhouse. The soils were deposited in $500 \mathrm{ml}$ plastic pots and planted with five sorghum seeds. Three weeks later, emerged sorghum seedlings were removed from the pots and their roots were gently shaken to collect the adhered soil. Serial dilution, plating and incubation conditions were conducted as described before for Ethiopian samples.

Bacteria also were isolated from the rhizoplane, i.e. those adhering to the surface of the roots by placing $1 \mathrm{~g}$ root in $9 \mathrm{ml} 1 \mathrm{M} \mathrm{MgSO}_{4}$ solution which was shaken manually for $1 \mathrm{~min}$. Ten fold serial dilutions of this solution were made after which a $0.1 \mathrm{ml}$ aliquot of the serially diluted suspension from each sample was plated on the two media described before. All plates were incubated at $28 \pm 2{ }^{\circ} \mathrm{C}$ for $24 \mathrm{~h}$. A total of 160 bacterial colonies randomly selected were 
preliminary characterized as described before and 86 isolates were chosen.

\section{Bacterial inoculum preparation}

Bacterial isolates were grown in nutrient broth (Biolab) on a rotary shaker (Labotech) at $28{ }^{\circ} \mathrm{C}$ for $24 \mathrm{~h}$. The suspension was centrifuged in $50 \mathrm{ml}$ capacity sterile plastic tubes at $5000 \mathrm{rpm}$ for $10 \mathrm{~min}$ using an Avanti TM J-25 Beckman centrifuge. The pellets were re-suspended in quarter strength sterile Ringer's (Merck) solution and the suspension was adjusted to give a final concentration of $10^{6}-10^{8} \mathrm{cfu} /$ $\mathrm{ml}\left(\mathrm{OD}_{550}=0.5-1 \cdot 5\right)$ using the viable plate count method and optical density measurement.

\section{Evaluation of bacterial isolates for growth promotion}

Prior to the greenhouse pot experiment, a preliminary screening of 78 bacterial isolates from Ethiopia and 86 isolates from South Africa was conducted for early root and shoot development in sorghum in 96 cavity styrofoam seedling trays filled with steam-pasteurized sandy loam topsoil. Sorghum seeds (Ethiopian variety, Meko) were surface sterilized with $70 \%$ ethanol $(736 \mathrm{ml} / \mathrm{l})$ for $5 \mathrm{~min}, 10 \mathrm{mg} / \mathrm{g}$ sodium hypochlorite in distilled water for $1 \mathrm{~min}$ and rinsed five times in sterile water. Three adjacent cavities in the tray, each planted with four seeds constituted one replicate. Therefore in each replicate, there were 12 plants in a completely randomized design. The bacterial inoculum $\left(30 \mathrm{ml}\right.$ of the $10^{6}-10^{8} \mathrm{cfu} / \mathrm{ml}$ suspension) was applied as a soil drench once a week for two weeks. The temperature of the greenhouse was maintained at $28{ }^{\circ} \mathrm{C}$ and watering was done twice daily regularly. The plants were harvested 3 weeks after the first inoculation. Shoot and root length as well as fresh and dry weight measurements were compared with the uninoculated control (data not shown).

Based on the results from the screening experiment in the seedling tray, 20 Ethiopian and 39 South African isolates were chosen for the greenhouse pot trial. Eight surface sterilized seeds were sown in a $120 \times 100 \mathrm{~mm}$ diameter pot containing steampasteurized topsoil. The seeds were watered regularly until the emergence of the first shoot. Five days later, $30 \mathrm{ml}$ of the bacterial inoculum was applied to the pots as a soil drench and a second application was made 1 week later. The pots were watered twice daily with an automatic watering system. The experiment was arranged in a complete randomized design with three replications and was repeated twice. Plants were harvested 35 days after planting and 30 days after inoculation. Growth promoting ability of the bacterial isolates was determined based on the data collected on shoot height, shoot dry and fresh weight, root length and root dry weight, leaf width and leaf chlorophyll content as measured with a Minolta
SPAD 502 chlorophyll meter (Minolta, Japan) and expressed in spad units.

\section{Root colonization}

Root colonization was assessed according to the procedure described by Han et al. (2000). The same plants and experimental layout used in the pot trial experiment described before were used when detecting root colonization by the bacterial isolates. Briefly, $1 \mathrm{~g}$ root per pot in each replication were surface sterilized as described before for sorghum seeds and macerated in $3 \mathrm{ml} 0 \cdot 1 \mathrm{M}$ Phosphate buffer ( $\mathrm{pH} 7$ ) using a homogenizer. The suspension was serially diluted in quarter strength Ringer's solution (Merck). Aliquots of $0.1 \mathrm{ml}$ were plated on Nutrient agar amended with $50 \mu \mathrm{g}$ rifampicin $/ \mathrm{ml}$. The plates were incubated at $28 \pm 2{ }^{\circ} \mathrm{C}$ for $24 \mathrm{~h}$. The number of colonies was recorded and root colonization expressed as $\mathrm{cfu} / \mathrm{g}$ root.

\section{Modes of action for growth promotion}

\section{Siderophore production}

Siderophore production was detected by the universal chemical assay using chrome-azurol S (CAS) agar (Schwyn \& Neilands 1987). Cultures of isolates that resulted in significant growth promotion in greenhouse pot experiments were grown in a modified minimal medium (under iron restricted and high iron condition) at $27 \pm 1{ }^{\circ} \mathrm{C}$ for $48 \mathrm{~h}$ and at $180 \mathrm{rpm}$ on a rotary shaker. Each culture was centrifuged at $10000 \mathrm{~g}$ for $10 \mathrm{~min}$ and the supernatant was collected. Three wells were made equidistantly on the CAS agar plate using $5 \mathrm{~mm}$ cork borer after which $30 \mu \mathrm{l}$ of the culture supernatant was deposited into the wells. Control plates received sterile broth media without bacteria under both low and high iron conditions. The plates were incubated at room temperature for $8 \mathrm{~h}$ after which any colour change in the medium was recorded.

\section{IAA production}

The production of IAA-like compounds was detected from the culture supernatants of the bacterial isolates selected based on their growth promoting efficiency following the procedure described by Thakuria et al. (2004). Briefly, pure colonies from a $24 \mathrm{~h}$ culture were inoculated into nutrient broth with $2 \mathrm{mg}$ trypthophan $/ \mathrm{g}$ of nutrient broth solution and in the absence of trypthophan, and were incubated at $28 \pm 2{ }^{\circ} \mathrm{C}$ for $48 \mathrm{~h}$. Five $\mathrm{ml}$ culture was removed from each tube and centrifuged at $10000 \mathrm{rpm}$ for $15 \mathrm{~min}$. Two millilitre aliquots of the supernatant were transferred to a fresh tube and washed with ethyl acetate to extract free IAA-like substance. The extractions were then treated with $4 \mathrm{ml}$ Salkowsky reagent $(1 \mathrm{ml} 0 \cdot 5 \mathrm{M} \mathrm{FeCl}$ 
in $50 \mathrm{ml} \mathrm{HClO}_{4}$ ) and incubated at room temperature for $25 \mathrm{~min}$. The absorbance of the solution (pink colour developed) was read at $530 \mathrm{~nm}$. For the control experiment, sterile nutrient broth was used. The concentration of IAA in the culture supernatants was determined using a calibration curve of pure IAA as a standard.

\section{Phosphate solubilization}

Phosphate solubilization activity of the bacterial isolates was detected by means of a plate assay using Pikovskaya (PVK) agar (Pikovskaya 1948) which results in a formation of a clear halo. PVK medium contained per litre: glucose, $10 \mathrm{~g}$; $\mathrm{Ca}_{3}\left(\mathrm{PO}_{4}\right)_{2}, 5 \mathrm{~g}$; $\left(\mathrm{NH}_{4}\right) \mathrm{SO}_{4}, 0.5 \mathrm{~g} ; \mathrm{NaCl}, 0.2 \mathrm{~g} ; \mathrm{MgSO}_{4} .7 \mathrm{H}_{2} \mathrm{O}, 0.1 \mathrm{~g}$; $\mathrm{KCl}, 0.2 \mathrm{~g} ; \mathrm{NaCl}, 0.2 \mathrm{~g} ; \mathrm{MnSO}_{4} \cdot \mathrm{H}_{2} \mathrm{O}, 0.002 \mathrm{~g}$; $\mathrm{FeSO}_{4} .7 \mathrm{H}_{2} \mathrm{O}, 0.002 \mathrm{~g}$; yeast extract, $0.5 \mathrm{~g}$. To compare the reproducibility of the halo formation, isolates were also tested on PVK agar supplemented with $1 \mathrm{mg} / \mathrm{l}$ bromophenol blue (Gupta et al. 1994) and a modified PVK medium devoid of yeast extract, i.e. NBRIY medium (Nautiyal 1999). A pure colony from a fresh culture of each isolate was stab inoculated in duplicate into each of the three-agar media using a sterile needle. Plates were incubated for 14 days at $28{ }^{\circ} \mathrm{C}$ after which the diameter of the clear halo was measured. Control plates were inoculated with sterile broth.

\section{Identification of bacterial isolates}

\section{Identification by API test strips}

Isolates were chosen and further identified to species level by means of the API identification system with the API Plus computer software (bioMèrieux). Grampositive, endospore forming rods were identified to the species using API ${ }^{\circledR} 50 \mathrm{CH}$ test strips. Gramnegative rod isolates with fermentative reaction in the Hugh \& Leifson $\mathrm{O} / \mathrm{F}$ test were identified using the API ${ }^{\circledR} 20$ E test strip while those with oxidative reaction were identified using the API 20 NE test strip.

\section{Identification by $16 S \mathrm{rDNA}$ sequencing}

Identification of isolates with less than 0.80 similarity of the species on the API data base was clarified by means of PCR amplification of the bacterial 16S rDNA. DNA from Gram-positive isolates was extracted using the DNeasy Tissue Kit (Qiagen) or from Gram-negative isolates, the boiling method (Mohran et al. 1998). A portion of the 16S rDNA gene (corresponding to positions 8-1541 in E. coli of each bacterial isolate was amplified using forward primer pA (5'-AGAGTTTGATCCTGGCTGAG-3') and reverse primer $\mathrm{pH}$ (5'-AAG GAG GTG ATC CAG CCG CA-3') (Coenye et al. 1999). PCR amplification was confirmed by size fractionation on a $10 \mathrm{mg} / \mathrm{g}$ agarose gel. Amplification products were sequenced using primer *pD (5'-CAG CAG CCG CGG TAA TAC-3') (Inqaba Biotech, South Africa). BLAST search of NCBI data libraries was used to establish the identification of the isolates.

\section{Statistical analysis}

The experiments were designed as completely randomized. One way analysis of variance (ANOVA) was applied to the data to test for differences between isolate effects using the statistical program Genstat ${ }^{\mathbb{R}}$ (Genstat Committee 2005). The data were mostly normally distributed but with heterogeneous treatment variances. Therefore significance was assessed at $P \leqslant 0 \cdot 10$. Fresh and dry shoot weight was square root transformed to stabilize isolate variances. Data on root colonization of the bacterial isolates were log transformed before subjecting to ANOVA. Based on the results of fresh and dry weight of shoots, the best set of isolates were grouped according to the Multiple $t$-distribution test procedure of Gupta \& Panchapakesan (1979) at the $P \leqslant 0 \cdot 99$. Pearson's linear correlation coefficient was used to evaluate the relationship between phosphate solubilization and root/shoot dry weight as well as the relationship between IAA concentration and root/shoot length.

\section{RESULTS}

\section{Greenhouse pot trial}

Three isolates from the rhizosphere of sorghum in Ethiopia resulted in a significant increase in at least two growth parameters. Two isolates (KBE5-1 and KBE7-8) resulted in a significant increase in at least five parameters (Table 1). Isolate KBE5-1 displayed increases in all parameters tested except chlorophyll content and root length. KBE7-8, on the other hand resulted in a significant increase in all seven parameters (Table 1; Fig. 1), the highest increases being in shoot and root biomass. According to the grouping of isolates using the multiple $t$-test distribution, 11 isolates resulted in an increase in shoot fresh weight. The highest increase was achieved by isolates KBE5-1 and KBE7-8 which increased shoot fresh weight by $2 \cdot 7$ and $3 \mathrm{~g}$ respectively over the uninoculated control $(0.63 \mathrm{~g})$. Compared with other less effective isolates, the two isolates increased shoot fresh weight between 2.5 and 2.75 g. Similarly only KBE5-1 and KBE7-8 were grouped together as the best isolates in terms of increase in shoot dry weight (Table 5). The increase due to inoculation with KBE7-8 ranged from $0 \cdot 4$ to $0.86 \mathrm{~g}$ compared to other isolates and the uninoculated control $(0 \cdot 306 \mathrm{~g})$. KBE5-1 similarly resulted in shoot dry weight increase of between 0.37 and $0.85 \mathrm{~g}$ (Table 5).

Among isolates obtained from the rhizosphere of grasses in South Africa, KBS9-B and NAS4-3 
Table 1. Inoculation of bacterial isolates from Ethiopia and their effect on shoot height, shoot fresh and dry weight, chlorophyll content, leaf width, root length and root dry weight of sorghum under greenhouse conditions.

Values are means \pm S.D.

\begin{tabular}{|c|c|c|c|c|c|c|c|}
\hline $\begin{array}{l}\text { Bacterial } \\
\text { isolates }\end{array}$ & $\begin{array}{c}\text { Shoot } \\
\text { height }(\mathrm{mm})\end{array}$ & $\begin{array}{l}\text { Shoot fresh } \\
\text { weight }(\mathrm{g})\end{array}$ & $\begin{array}{l}\text { Shoot dry } \\
\text { weight }(g)\end{array}$ & $\begin{array}{l}\text { Chlorophyll } \\
\text { content } \\
\text { (spad units) }\end{array}$ & $\begin{array}{c}\text { Leaf } \\
\text { width }(\mathrm{mm})\end{array}$ & $\begin{array}{c}\text { Root } \\
\text { length }(\mathrm{mm})\end{array}$ & $\begin{array}{l}\text { Root dry } \\
\text { mass (g) }\end{array}$ \\
\hline KBE4-3 & $195 \pm 9 \cdot 6$ & $1 \cdot 5 \pm 0 \cdot 080$ & $0 \cdot 16 \pm 0 \cdot 030$ & $21 \cdot 4 \pm 2 \cdot 68$ & $3 \cdot 9 \pm 0 \cdot 23$ & $128 \pm 55 \cdot 5$ & $0 \cdot 07 \pm 0.015$ \\
\hline KBE5-3 & $229 \pm 27 \cdot 3$ & $2 \cdot 17 \pm 0 \cdot 250$ & $0 \cdot 27 \pm 0 \cdot 061$ & $21 \cdot 6 \pm 0 \cdot 55$ & $4 \cdot 4 \pm 0 \cdot 20$ & $160 \pm 31 \cdot 0$ & $0 \cdot 08 \pm 0 \cdot 015$ \\
\hline KBE5-7 & $235 \pm 5.87$ & $2 \cdot 25 \pm 0 \cdot 317$ & $0 \cdot 33 \pm 0 \cdot 226$ & $21 \cdot 8 \pm 2 \cdot 64$ & $4 \cdot 2 \pm 0 \cdot 40$ & $143 \pm 16 \cdot 6$ & $0 \cdot 01 \pm 0 \cdot 000$ \\
\hline NAE4-1 & $216 \pm 2 \cdot 26$ & $1 \cdot 73 \pm 0 \cdot 154$ & $0 \cdot 20 \pm 0 \cdot 021$ & $21 \cdot 8 \pm 2 \cdot 76$ & $4 \cdot 4 \pm 0 \cdot 50$ & $193 \pm 26 \cdot 4$ & $0 \cdot 06 \pm 0 \cdot 010$ \\
\hline NAE7-1 & $210 \pm 43 \cdot 8$ & $2 \cdot 08 \pm 0 \cdot 268$ & $0 \cdot 30 \pm 0 \cdot 040$ & $22 \cdot 5 \pm 8 \cdot 87$ & $4 \cdot 2 \pm 0 \cdot 50$ & $173 \pm 16 \cdot 7$ & $0.06 \pm 0.019$ \\
\hline KBE7-6 & $211 \pm 11 \cdot 5$ & $1 \cdot 97 \pm 0 \cdot 144$ & $0 \cdot 22 \pm 0 \cdot 080$ & $22 \cdot 7 \pm 12 \cdot 2$ & $4 \cdot 2 \pm 0 \cdot 34$ & $169 \pm 51 \cdot 1$ & $0 \cdot 05 \pm 0.035$ \\
\hline KBE5-2 & $195 \pm 37 \cdot 0$ & $1 \cdot 17 \pm 0.075$ & $0 \cdot 17 \pm 0 \cdot 055$ & $17 \cdot 0 \pm 12 \cdot 8$ & $4 \cdot 8 \pm 0 \cdot 34$ & $119 \pm 20 \cdot 0$ & $0.04 \pm 0.039$ \\
\hline KBE9-4 & $288 \pm 124 \cdot 0$ & $4 \cdot 52 \pm 0 \cdot 230$ & $0 \cdot 66 \pm 0 \cdot 148$ & $23 \cdot 8 \pm 5 \cdot 33$ & $8 \cdot 2 \pm 5 \cdot 20$ & $138 \pm 5 \cdot 3$ & $0 \cdot 10 \pm 0 \cdot 264$ \\
\hline KBE9-1 & $353 \pm 17 \cdot 5$ & $4 \cdot 09 \pm 1 \cdot 417$ & $0 \cdot 50 \pm 0.095$ & $24 \cdot 8 \pm 2.95$ & $7 \cdot 0 \pm 4 \cdot 38$ & $263 \pm 47 \cdot 5$ & $0.08 \pm 0.005$ \\
\hline NAE2-8 & $232 \pm 20 \cdot 7$ & $1 \cdot 71 \pm 0.546$ & $0 \cdot 21 \pm 0 \cdot 011$ & $24 \cdot 4 \pm 1 \cdot 87$ & $6 \cdot 5 \pm 1 \cdot 00$ & $149 \pm 24 \cdot 4$ & $0.03 \pm 0.012$ \\
\hline KBE8-2 & $217 \pm 14 \cdot 0$ & $1 \cdot 33 \pm 0 \cdot 200$ & $0 \cdot 23 \pm 0 \cdot 055$ & $24 \cdot 4 \pm 2 \cdot 36$ & $5 \cdot 2 \pm 0 \cdot 40$ & $174 \pm 8 \cdot 7$ & $0.06 \pm 0.017$ \\
\hline KBE6-3 & $306 \pm 103 \cdot 0$ & $1 \cdot 55 \pm 0 \cdot 360$ & $0 \cdot 13 \pm 0 \cdot 100$ & $25 \cdot 3 \pm 0 \cdot 90$ & $5 \cdot 9 \pm 0.46$ & $134 \pm 19 \cdot 1$ & $0 \cdot 05 \pm 0.030$ \\
\hline NAE9-5 & $232 \pm 49 \cdot 3$ & $1.65 \pm 0.670$ & $0 \cdot 26 \pm 0.079$ & $25 \cdot 3 \pm 0 \cdot 20$ & $5 \cdot 4 \pm 0 \cdot 70$ & $187 \pm 11 \cdot 4$ & $0.04 \pm 0.025$ \\
\hline KBE5-1 & $384 \pm 61 \cdot 0$ & $12 \cdot 4 \pm 0 \cdot 426$ & $1 \cdot 33 \pm 0.057$ & $26 \cdot 2 \pm 6 \cdot 11$ & $9 \cdot 3 \pm 4 \cdot 30$ & $244 \pm 2 \cdot 3$ & $0 \cdot 11 \pm 0 \cdot 012$ \\
\hline KBE5-8 & $250 \pm 69 \cdot 4$ & $7 \cdot 70 \pm 3 \cdot 980$ & $0.59 \pm 0.425$ & $28 \cdot 4 \pm 3 \cdot 75$ & $9 \cdot 2 \pm 4 \cdot 01$ & $128 \pm 28 \cdot 0$ & $0.08 \pm 0.264$ \\
\hline KBE7-8 & $369 \pm 67 \cdot 1$ & $14 \cdot 84 \pm 3 \cdot 770$ & $1.41 \pm 0.601$ & $29 \cdot 8 \pm 13 \cdot 9$ & $12 \cdot 8 \pm 1 \cdot 90$ & $296 \pm 5 \cdot 0$ & $0 \cdot 13 \pm 0 \cdot 264$ \\
\hline NAE5-7 & $177 \pm 14 \cdot 3$ & $1 \cdot 78 \pm 0.194$ & $0 \cdot 22 \pm 0.052$ & $19 \cdot 4 \pm 3 \cdot 14$ & $3.9 \pm 0.65$ & $145 \pm 18 \cdot 6$ & $0.07 \pm 0.020$ \\
\hline KBE1-7 & $203 \pm 16 \cdot 6$ & $1 \cdot 39 \pm 0.083$ & $0 \cdot 19 \pm 0.036$ & $18 \cdot 5 \pm 2 \cdot 55$ & $4 \cdot 0 \pm 0 \cdot 77$ & $147 \pm 20 \cdot 0$ & $0.06 \pm 0.010$ \\
\hline KBE8-3 & $154 \pm 6.63$ & $1 \cdot 43 \pm 0 \cdot 354$ & $0 \cdot 17 \pm 0 \cdot 002$ & $18 \cdot 2 \pm 3 \cdot 78$ & $4 \cdot 0 \pm 0 \cdot 11$ & $179 \pm 11 \cdot 0$ & $0.04 \pm 0.028$ \\
\hline KBE6-1 & $223 \pm 34 \cdot 9$ & $2 \cdot 08 \pm 0.466$ & $0 \cdot 27 \pm 0 \cdot 062$ & $20 \cdot 4 \pm 1 \cdot 67$ & $4 \cdot 2 \pm 1 \cdot 62$ & $193 \pm 11 \cdot 4$ & $0.09 \pm 0.012$ \\
\hline Control & $181 \pm 1 \cdot 47$ & $0.63 \pm 0.340$ & $0 \cdot 10 \pm 0 \cdot 062$ & $19 \cdot 9 \pm 4 \cdot 71$ & $3 \cdot 0 \pm 0 \cdot 60$ & $94 \pm 8 \cdot 5$ & $0.008 \pm 0.008$ \\
\hline S.E.D. ${ }^{*}$ & $40 \cdot 84$ & $0 \cdot 2014$ & $0 \cdot 1080$ & $4 \cdot 924$ & $1 \cdot 704$ & $20 \cdot 82$ & $0 \cdot 017$ \\
\hline D.F. & 42 & 42 & 42 & 42 & 42 & 42 & 42 \\
\hline$P$ & $P<0.001$ & $P<0.001$ & $P<0.001$ & NS & $P<0.001$ & $P<0.001$ & $P<0.001$ \\
\hline
\end{tabular}

* S.E.D. is the standard error of difference.

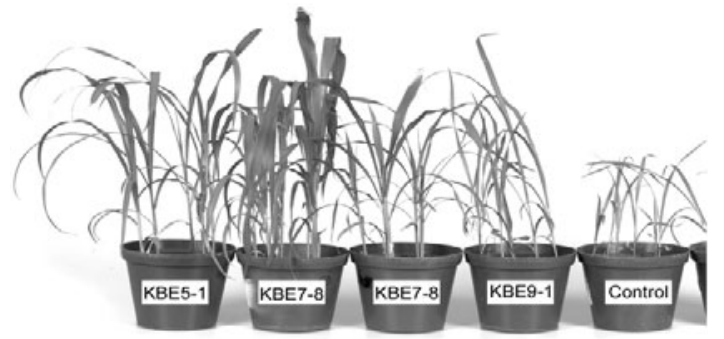

Fig. 1. Shoot growth enhancement in five weeks old sorghum plants by rhizobacterial isolates from Ethiopia. Plants in the control treatment showed retarded growth in comparison with plants treated with bacterial isolates Bacillus cereus strains KBE5-1, KBE7-8 and KBE9-1.

resulted in significant increases in all seven parameters measured (Table 2; Fig. 2). Another isolate (KBS5-H) also rendered significant increases in shoot and root length (Table 2; Fig. 2). This isolate increased shoot and root length significantly but it had less effect on shoot and root dry weights than isolates KBS9-B and NAS4-3. Other isolates which stimulated growth in sorghum were isolates KBS6-H, KBS5-F, KBS2-12 and KFP9-E all of which resulted in significant increases in five parameters. The remaining isolates resulted in significant increases in at least three parameters except for isolates KBS1-T and KBS10-E which resulted in increases in two parameters. Most of the isolates which resulted in significant growth promotion mainly affected plant biomass (fresh and dry weights of shoots and roots), root length and chlorophyll content. Compared to the control, these isolates had no effect on shoot length and leaf width.

Five isolates viz. KFP9-E, KBS5-F, KBS6-H, KBS9-B and NAS4-3 have been grouped as the best performing isolates in terms of increase in fresh and dry weight of the shoots, the highest being by KBS9-B and NAS4-3 (Table 5). The increase in shoot fresh weight ranged from 5 to $9 \mathrm{~g}$ for NAS4-3 and from 3 to $6.7 \mathrm{~g}$ for KBS9-B compared to other isolates and the control (0.623 g) (Table 5). These two isolates and another isolate KBS6-H also resulted in the highest 
Table 2. Inoculation of bacterial isolates from South Africa and their effect on shoot height, shoot fresh and dry weight, chlorophyll content, leaf width, root length and root dry weight of sorghum under greenhouse conditions. Values are means \pm S.D.

\begin{tabular}{|c|c|c|c|c|c|c|c|}
\hline $\begin{array}{l}\text { Bacterial } \\
\text { isolates }\end{array}$ & $\begin{array}{c}\text { Shoot } \\
\text { height }(\mathrm{mm})\end{array}$ & $\begin{array}{c}\text { Shoot fresh } \\
\text { weight }(\mathrm{g})\end{array}$ & $\begin{array}{l}\text { Shoot dry } \\
\text { weight }(g)\end{array}$ & $\begin{array}{l}\text { Chlorophyll } \\
\text { (spad units) }\end{array}$ & $\begin{array}{c}\text { Leaf } \\
\text { width }(\mathrm{mm})\end{array}$ & $\begin{array}{c}\text { Root } \\
\text { length }(\mathrm{mm})\end{array}$ & $\begin{array}{c}\text { Root dry } \\
\text { weight (g) }\end{array}$ \\
\hline KBS9-B & $415 \pm 77 \cdot 2$ & $7 \cdot 4 \pm 3 \cdot 19$ & $1 \cdot 8 \pm 0 \cdot 16$ & $30 \cdot 0 \pm 2 \cdot 21$ & $12 \cdot 7 \pm 2 \cdot 85$ & $216 \pm 8 \cdot 0$ & $0 \cdot 25 \pm 0 \cdot 025$ \\
\hline NAS4-3 & $406 \pm 46 \cdot 2$ & $9 \cdot 4 \pm 3 \cdot 50$ & $1 \cdot 4 \pm 0 \cdot 13$ & $28 \cdot 3 \pm 1 \cdot 10$ & $11 \cdot 4 \pm 3 \cdot 22$ & $209 \pm 14 \cdot 0$ & $0 \cdot 28 \pm 0 \cdot 135$ \\
\hline KBS1-F & $305 \pm 54 \cdot 0$ & $4 \cdot 0 \pm 1 \cdot 82$ & $0 \cdot 7 \pm 0 \cdot 19$ & $20 \cdot 2 \pm 1 \cdot 02$ & $6 \cdot 6 \pm 1 \cdot 33$ & $58 \pm 6 \cdot 4$ & $0 \cdot 16 \pm 0 \cdot 042$ \\
\hline KBS5-F & $305 \pm 1 \cdot 4$ & $6 \cdot 8 \pm 1 \cdot 56$ & $1 \cdot 0 \pm 0 \cdot 10$ & $24.8 \pm 1.97$ & $6 \cdot 5 \pm 0.49$ & $198 \pm 27 \cdot 5$ & $0 \cdot 20 \pm 0 \cdot 020$ \\
\hline KBS2-12 & $303 \pm 44 \cdot 4$ & $3 \cdot 9 \pm 0 \cdot 85$ & $0 \cdot 7 \pm 0 \cdot 11$ & $26 \cdot 8 \pm 0 \cdot 90$ & $7 \cdot 2 \pm 0.66$ & $212 \pm 13 \cdot 6$ & $0.25 \pm 0.277$ \\
\hline KBS9-H & $297 \pm 44 \cdot 6$ & $4 \cdot 3 \pm 1 \cdot 70$ & $0 \cdot 5 \pm 0 \cdot 19$ & $29 \cdot 2 \pm 1 \cdot 49$ & $7 \cdot 3 \pm 0 \cdot 43$ & $215 \pm 13 \cdot 2$ & $0 \cdot 10 \pm 0 \cdot 020$ \\
\hline KFP9-K & $289 \pm 32 \cdot 3$ & $2 \cdot 9 \pm 0 \cdot 32$ & $0.6 \pm 0.06$ & $28 \cdot 4 \pm 1 \cdot 40$ & $7 \cdot 5 \pm 0.89$ & $210 \pm 19 \cdot 0$ & $0 \cdot 10 \pm 0.006$ \\
\hline KBS5-H & $435 \pm 67 \cdot 2$ & $11.9 \pm 0.83$ & $0 \cdot 6 \pm 0 \cdot 17$ & $30 \cdot 3 \pm 2 \cdot 06$ & $7 \cdot 8 \pm 1 \cdot 72$ & $241 \pm 7 \cdot 2$ & $0.08 \pm 0.006$ \\
\hline KFP9-E & $285 \pm 99 \cdot 0$ & $5 \cdot 5 \pm 3 \cdot 21$ & $1 \cdot 0 \pm 0.27$ & $24 \cdot 3 \pm 0 \cdot 44$ & $4 \cdot 5 \pm 3 \cdot 01$ & $115 \pm 10 \cdot 0$ & $0 \cdot 21 \pm 0 \cdot 035$ \\
\hline KBS6-1 & $283 \pm 33 \cdot 5$ & $3 \cdot 0 \pm 0 \cdot 15$ & $0.8 \pm 0.04$ & $23 \cdot 4 \pm 3 \cdot 87$ & $6 \cdot 1 \pm 0 \cdot 32$ & $155 \pm 11 \cdot 5$ & $0 \cdot 10 \pm 0 \cdot 0001$ \\
\hline KBS6-H & $282 \pm 77 \cdot 2$ & $7 \cdot 0 \pm 2 \cdot 43$ & $1 \cdot 7 \pm 0 \cdot 18$ & $30 \cdot 5 \pm 1 \cdot 94$ & $10 \cdot 0 \pm 2 \cdot 85$ & $206 \pm 15 \cdot 5$ & $0 \cdot 13 \pm 0.066$ \\
\hline NAS2-B & $269 \pm 36 \cdot 9$ & $4 \cdot 1 \pm 0.66$ & $0.5 \pm 0.45$ & $24 \cdot 5 \pm 1 \cdot 30$ & $6 \cdot 0 \pm 1 \cdot 05$ & $83 \pm 13 \cdot 6$ & $0 \cdot 08 \pm 0.021$ \\
\hline NAS1-6 & $258 \pm 15 \cdot 3$ & $3.9 \pm 0.95$ & $0 \cdot 7 \pm 0 \cdot 21$ & $20 \cdot 2 \pm 2 \cdot 09$ & $7 \cdot 0 \pm 1 \cdot 88$ & $83 \pm 23 \cdot 6$ & $0.09 \pm 0.059$ \\
\hline NAS6-N & $256 \pm 58 \cdot 5$ & $4 \cdot 2 \pm 1 \cdot 36$ & $0.6 \pm 0.26$ & $19 \cdot 7 \pm 1 \cdot 01$ & $6 \cdot 3 \pm 8 \cdot 01$ & $198 \pm 13 \cdot 2$ & $0 \cdot 10 \pm 0 \cdot 041$ \\
\hline KBS1-J & $252 \pm 31 \cdot 3$ & $3 \cdot 7 \pm 0.08$ & $0 \cdot 5 \pm 0 \cdot 13$ & $23 \cdot 4 \pm 3 \cdot 15$ & $6 \cdot 3 \pm 0 \cdot 28$ & $75 \pm 15 \cdot 6$ & $0.09 \pm 0.029$ \\
\hline KBS10-E & $248 \pm 10 \cdot 0$ & $1 \cdot 5 \pm 0 \cdot 30$ & $0 \cdot 4 \pm 0 \cdot 17$ & $23 \cdot 3 \pm 2 \cdot 50$ & $4 \cdot 7 \pm 1 \cdot 38$ & $87 \pm 17 \cdot 6$ & $0 \cdot 10 \pm 0 \cdot 046$ \\
\hline KBS6-17 & $225 \pm 31 \cdot 9$ & $3 \cdot 8 \pm 0 \cdot 74$ & $0 \cdot 4 \pm 0 \cdot 12$ & $19 \cdot 7 \pm 1 \cdot 78$ & $6 \cdot 8 \pm 0 \cdot 25$ & $71 \pm 23 \cdot 2$ & $0.09 \pm 0.036$ \\
\hline KBS6-11 & $238 \pm 55 \cdot 7$ & $2 \cdot 8 \pm 1 \cdot 36$ & $0 \cdot 6 \pm 0 \cdot 10$ & $20 \cdot 9 \pm 1.73$ & $6 \cdot 3 \pm 0 \cdot 58$ & $183 \pm 25 \cdot 5$ & $0.13 \pm 0.023$ \\
\hline KBS1-T & $237 \pm 45 \cdot 1$ & $3 \cdot 2 \pm 1 \cdot 35$ & $0 \cdot 5 \pm 0 \cdot 12$ & $18 \cdot 6 \pm 2 \cdot 48$ & $6 \cdot 3 \pm 0 \cdot 47$ & $200 \pm 15 \cdot 0$ & $0.09 \pm 0.012$ \\
\hline KBS9-R & $235 \pm 38 \cdot 0$ & $3 \cdot 1 \pm 0 \cdot 39$ & $0 \cdot 4 \pm 0 \cdot 12$ & $19 \cdot 8 \pm 0 \cdot 79$ & $7 \cdot 0 \pm 1 \cdot 21$ & $77 \pm 7 \cdot 4$ & $0.08 \pm 0.006$ \\
\hline Control & $147 \pm 38 \cdot 9$ & $0 \cdot 6 \pm 0 \cdot 45$ & $0 \cdot 5 \pm 0 \cdot 03$ & $16 \cdot 8 \pm 0 \cdot 70$ & $5 \cdot 6 \pm 0 \cdot 26$ & $57 \pm 5.9$ & $0 \cdot 02 \pm 0 \cdot 021$ \\
\hline S.E.D.* & $38 \cdot 54$ & $13 \cdot 49$ & $1 \cdot 50$ & $15 \cdot 50$ & $1 \cdot 19$ & $12 \cdot 9$ & $0 \cdot 605$ \\
\hline D.F. & 42 & 42 & 42 & 42 & 42 & 42 & 42 \\
\hline$P$ & $P<0.001$ & $P<0.001$ & $P<0.001$ & $P<0.001$ & $P<0.001$ & $P<0.001$ & $P=0.004$ \\
\hline
\end{tabular}

* S.E.D. is the standard error of difference.

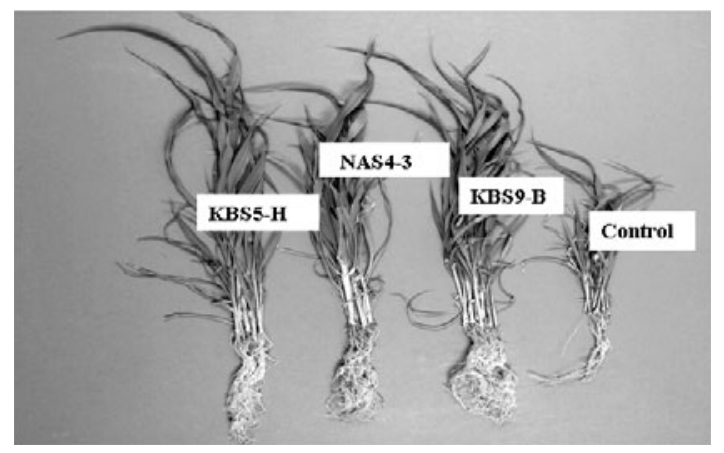

Fig. 2. Shoot and root growth enhancement in five weeks old sorghum plants by $B$. cereus KBS5-H and NAS4-3 and Stenotrophomonas maltophilia KBS9-B isolated from the rhizosphere of grasses at Nylsvlei Nature Reserve in South Africa in comparison with the un-inoculated control.

increase in shoot dry weight. The highest increase in this case was obtained with KBS9-B ranging from $0 \cdot 8$ to $1.75 \mathrm{~g}$. The shoot dry weight of the control was $0 \cdot 0467 \mathrm{~g}$. Isolates which were antagonistic to growth in the preliminary screening of this experiment (data not shown) were not used in the subsequent experiments.

\section{Root colonization}

All the initially applied bacterial isolates grew on the rifampicin augmented medium and were different from the control plates both morphologically and quantitatively. Only very few colonies which probably escaped the steam pasteurization process were retrieved on the control plates. Both Ethiopian and South African isolates, which resulted in significant growth promotion in sorghum, were able to colonize roots successfully. Six isolates from the rhizosphere of grasses in South Africa and five isolates from sorghum rhizosphere in Ethiopia colonized sorghum roots with a concentration of $\geqslant 10^{5} \mathrm{cfu} / \mathrm{g}$ root. The count of bacterial colonies similar to the inoculated ones for each of the isolates KBS9-B, KFP9-K, KBS9-H, KBS6-H, KBS5-H and NAS4-3 was $10^{8} \mathrm{cfu} / \mathrm{g}$ compared to the uninoculated control which had only $\leqslant 10^{4} \mathrm{cfu} / \mathrm{g}$ (Table 3). Similarly Ethiopian isolates KBE7-8, KBE5-1, KBE9-1, KBE5-7 and 
Table 3. Origin of rhizobacterial isolates from Ethiopia and South Africa and their ability to colonize sorghum roots under greenhouse conditions. Values are means \pm S.D.

\begin{tabular}{|c|c|c|c|c|c|c|}
\hline \multicolumn{4}{|c|}{ South African isolates (Grass rhizosphere) } & \multicolumn{3}{|c|}{ Ethiopian isolates (Sorghum rhizosphere) } \\
\hline Isolate & Log cfu/g士s.D. & Origin & Host grass & Isolate & Log cfu/g \pm S.D. & Origin \\
\hline KBS9-B & $8 \cdot 30 \pm 0 \cdot 085$ & Rhizoplane & Cyprus esculentus L. & KBE7-8 & $8 \cdot 19 \pm 0 \cdot 040$ & Rhizosphere \\
\hline KFP9-K & $8 \cdot 19 \pm 0 \cdot 098$ & Rhizoplane & C. esculentus $\mathrm{L}$. & KBE5-1 & $8 \cdot 09 \pm 0.035$ & Rhizosphere \\
\hline KBS9-H & $8 \cdot 17 \pm 0 \cdot 072$ & Rhizoplane & C. esculentus $\mathrm{L}$. & KBE9-1 & $8 \cdot 08 \pm 0 \cdot 071$ & Rhizosphere \\
\hline KBS6-H & $8 \cdot 16 \pm 0 \cdot 086$ & Rhizoplane & $\begin{array}{l}\text { Arstidia canescens subsp. } \\
\text { canescens }\end{array}$ & KBE5-7 & $8 \cdot 07 \pm 0 \cdot 130$ & Rhizosphere \\
\hline KBS5-H & $8 \cdot 08 \pm 0 \cdot 040$ & Rhizoplane & Eragrostis biflora & KBE5-8 & $8 \cdot 06 \pm 0 \cdot 046$ & Rhizosphere \\
\hline NAS4-3 & $8 \cdot 03 \pm 0 \cdot 040$ & Rhizosphere & Themeda triandra & KBE7-6 & $7 \cdot 03 \pm 0 \cdot 050$ & Rhizosphere \\
\hline KBS6-11 & $7 \cdot 22 \pm 0 \cdot 138$ & Rhizosphere & $\begin{array}{l}\text { A. canescens subsp. } \\
\text { canescens }\end{array}$ & KBE4-3 & $6 \cdot 56 \pm 1 \cdot 227$ & Rhizosphere \\
\hline KBS2-12 & $7 \cdot 06 \pm 0 \cdot 027$ & Rhizosphere & $\begin{array}{l}\text { Stipagrotis zeyheri subsp. } \\
\text { sericans }\end{array}$ & NAE7-1 & $6 \cdot 06 \pm 0 \cdot 046$ & Rhizosphere \\
\hline KBS6-1 & $6 \cdot 98 \pm 0 \cdot 017$ & Rhizosphere & $\begin{array}{l}\text { A. canescens subsp. } \\
\text { canescens }\end{array}$ & KBE6-1 & $5 \cdot 99 \pm 0 \cdot 011$ & Rhizosphere \\
\hline KBS1-T & $6 \cdot 61 \pm 0.532$ & Rhizoplane & Sporobolus fimbriatus & KBE6-3 & $5 \cdot 97 \pm 0 \cdot 030$ & Rhizosphere \\
\hline NAS6-N & $5 \cdot 94 \pm 0 \cdot 513$ & Rhizoplane & $\begin{array}{l}\text { A. canescens subsp. } \\
\text { canescens }\end{array}$ & NAE4-1 & $5 \cdot 93 \pm 0 \cdot 047$ & Rhizosphere \\
\hline KFP9-E & $5 \cdot 83 \pm 0 \cdot 142$ & Rhizoplane & C. esculentus $\mathrm{L}$. & KBE5-2 & $5 \cdot 90 \pm 0 \cdot 044$ & Rhizosphere \\
\hline NAS2-B & $5 \cdot 76 \pm 0 \cdot 126$ & Rhizoplane & $\begin{array}{l}\text { S. zeyheri subsp. } \\
\text { sericans }\end{array}$ & KBE8-2 & $5 \cdot 84 \pm 0 \cdot 066$ & Rhizosphere \\
\hline KBS2-3 & $4 \cdot 97 \pm 0 \cdot 015$ & Rhizosphere & $\begin{array}{l}\text { S. zeyheri subsp. } \\
\text { sericans }\end{array}$ & NAE2-8 & $4 \cdot 88 \pm 0 \cdot 021$ & Rhizosphere \\
\hline NAS1-6 & $4 \cdot 92 \pm 0 \cdot 011$ & Rhizosphere & S. fimbriatus & KBE9-4 & $4 \cdot 88 \pm 0 \cdot 057$ & Rhizosphere \\
\hline KBS1-F & $4 \cdot 88 \pm 0 \cdot 055$ & Rhizoplane & S. fimbriatus & Control & $4 \cdot 32 \pm 0 \cdot 195$ & - \\
\hline KBS1-J & $4 \cdot 84 \pm 0 \cdot 034$ & Rhizoplane & S. fimbriatus & & & \\
\hline KBS10-E & $4 \cdot 77 \pm 0 \cdot 035$ & Rhizoplane & C. esculentus L. & & & \\
\hline Control & $4 \cdot 44 \pm 0 \cdot 065$ & & - & & & \\
\hline S.E.D. ${ }^{*}$ & $0 \cdot \overline{11} 62$ & - & - & S.E.D. ${ }^{*}$ & $0 \cdot 2573$ & \\
\hline D.F. & 38 & - & - & D.F. & 32 & \\
\hline$P$ & $P<0.001$ & - & - & $P$ & $P<0.001$ & \\
\hline
\end{tabular}

* S.E.D. is the standard error of difference.

KBE5-8 were detected at levels $\geqslant 10^{8} \mathrm{cfu} / \mathrm{g}$ root. Most of the other isolates were however detected at a lower level than the initial inoculum level of $10^{8}-10^{9} \mathrm{cfu} / \mathrm{g}$ and the decrease from the initial inoculum level ranged between $10^{1}$ and $10^{4} \mathrm{cfu} / \mathrm{g}$.

\section{Modes of action for growth promotion}

\section{Siderophore production}

Five bacterial isolates, two from sorghum rhizosphere and three from the rhizosphere of grasses, were able to produce siderophores on CAS agar plates (Table 4). This was confirmed by a change in the colour of the CAS agar plates from blue to orange/yellow as a result of the siderophores sequestering and binding iron from the medium. Only one of the two isolates from the rhizosphere of sorghum in Ethiopia that produced siderophores in culture (KBE9-1) enhanced growth as measured by a significant increase in root length and root dry weight. On the other hand, of all the isolates from the rhizosphere of grasses obtained within South Africa, only isolates KBS6-H, KBS5-F, KBS9$\mathrm{R}$ and KBS6-17 tested positive for the production of siderophores on CAS agar plates (Table 4). Isolates KBS9-R and KBS6-17 did not however result in any significant increase in the growth of sorghum according to the parameters measured in this study (Tables 1 and 2).

\section{IAA production}

Of the 15 isolates tested for the production of the hormone IAA, 11 (a proportion of 0.73 ) were capable of producing the hormone in liquid culture with concentrations ranging between 4.2 and $22.8 \mu \mathrm{g} / \mathrm{ml}$ in the presence of $2 \mathrm{mg}$ tryptophan/g of nutrient broth solution (Table 4). This concentration however decreased significantly in the absence of tryptophan ranging between 1.82 and $5.43 \mu \mathrm{g} / \mathrm{ml}$ IAA. The 
Table 4. Measurements of siderophore production, phosphate solubilization and IAA production by selected bacterial isolates from the sorghum and grasses rhizosphere. Values are means \pm S.D.

\begin{tabular}{|c|c|c|c|c|c|c|}
\hline \multirow[b]{2}{*}{$\begin{array}{l}\text { Bacterial } \\
\text { isolate }\end{array}$} & \multicolumn{2}{|c|}{ Siderophore production* } & \multicolumn{2}{|c|}{ Phosphate solubilization $\dagger$} & \multicolumn{2}{|c|}{ IAA $(\mu \mathrm{g} / \mathrm{ml})$} \\
\hline & $\begin{array}{l}\text { High } \\
\text { iron }\end{array}$ & $\begin{array}{l}\text { Low } \\
\text { iron }\end{array}$ & $\begin{array}{l}\text { Pikovskaya } \\
\text { agar }\end{array}$ & $\begin{array}{l}\text { NBRIY } \\
\text { medium }\end{array}$ & $\begin{array}{l}2 \mathrm{mg} \text { tryptophan/g } \\
\text { nutrient broth solution }\end{array}$ & $\begin{array}{l}\text { No } \\
\text { tryptophan }\end{array}$ \\
\hline KBS6-11 & - & - & - & - & $10 \cdot 4 \pm 2 \cdot 19$ & $2 \cdot 6 \pm 0 \cdot 89$ \\
\hline KBS6-H & + & ++ & ++++ & +++++ & $21 \cdot 4 \pm 3 \cdot 00$ & $5 \cdot 4 \pm 5 \cdot 70$ \\
\hline KFP9-K & - & - & + & + & 0.00 & $0 \cdot 00$ \\
\hline KBS5-H & - & - & +++ & + & $22 \cdot 8 \pm 2 \cdot 82$ & $2 \cdot 2 \pm 0 \cdot 47$ \\
\hline KBE5-1 & - & - & - & - & $10 \cdot 6 \pm 2 \cdot 12$ & $2 \cdot 7 \pm 1 \cdot 47$ \\
\hline NAS4-3 & - & - & + & - & $20 \cdot 5 \pm 2 \cdot 17$ & $3 \cdot 4 \pm 3 \cdot 22$ \\
\hline KBS9-H & - & - & ++ & - & $22 \cdot 6 \pm 5 \cdot 20$ & $5 \cdot 2 \pm 3 \cdot 84$ \\
\hline KBS9-R & ++ & +++ & ++ & +++ & $4 \cdot 2 \pm 2 \cdot 83$ & $2 \cdot 1 \pm 1 \cdot 36$ \\
\hline KBE7-8 & - & - & ++ & ++++ & $12 \cdot 4 \pm 3 \cdot 82$ & $2 \cdot 1 \pm 1 \cdot 74$ \\
\hline KBS9-B & - & - & + & + & $15 \cdot 5 \pm 0 \cdot 89$ & $2 \cdot 3 \pm 2 \cdot 01$ \\
\hline KBS1-T & - & - & ++ & - & 0.00 & $0 \cdot 00$ \\
\hline KBE9-1 & ++ & +++ & ++++ & +++++ & $20 \cdot 8 \pm 5 \cdot 86$ & $2 \cdot 2 \pm 0 \cdot 48$ \\
\hline KBS5-F & + & ++ & ++++ & +++++ & 0.00 & $0 \cdot 00$ \\
\hline NAE5-7 & + & ++ & - & - & $7 \cdot 5 \pm 2 \cdot 63$ & $1 \cdot 8 \pm 0 \cdot 19$ \\
\hline Control & - & - & - & - & $0 \cdot 00$ & $0 \cdot 00$ \\
\hline S.E.D.t & - & - & - & - & $2 \cdot 33$ & $1 \cdot 71$ \\
\hline D.F. & - & - & - & - & 30 & 30 \\
\hline$P$ & - & - & - & - & $P<0.001$ & $P=0.066$ \\
\hline
\end{tabular}

* Siderophore production was compared among the bacterial isolates by measuring the diameter of yellow/orange halo produced on CAS agar plates: $+=$ halo diameter $\leqslant 3 \mathrm{~mm},++=3-5 \mathrm{~mm},+++=>5 \mathrm{~mm}$.

$\dagger$ Diameter of clear zone formed around the bacterial colony as a result of solubilization of tri-calcium phosphate on Pikovskaya and NBRIY agar medium: $+=\leqslant 3 \mathrm{~mm}$ clear zone, $++=3-5 \mathrm{~mm}$ clear zone, $+++=5-8 \mathrm{~mm}$ clear zone, $++++=8-10 \mathrm{~mm}$ clear zone, $+++++=>10 \mathrm{~mm}$ clear zone.

$\$$ S.E.D. is the standard error of difference.

highest amount of IAA was produced by isolate KBS5-H $(22 \cdot 8 \mu \mathrm{g} / \mathrm{ml})$ followed by isolates KBS9-H $(22.6 \mu \mathrm{g} / \mathrm{ml})$, KBS6-H $(21 \cdot 4 \mu \mathrm{g} / \mathrm{ml})$, KBE9-1 $(20 \cdot 8 \mu \mathrm{g} /$ $\mathrm{ml})$ and NAS4-3 $(20.5 \mu \mathrm{g} / \mathrm{ml})$ in the presence of tryptophan. In the absence of tryptophan the amount produced by these isolates decreased to $2 \cdot 24,5 \cdot 21$, $5 \cdot 43,2 \cdot 17,3 \cdot 4 \mu \mathrm{g} / \mathrm{ml}$ respectively. Isolates KBS5-F, KFP9-K and KBS1-T all of which affected some aspect of sorghum growth under greenhouse condition (Table 2) were unable to produce IAA in culture (Table 4).

\section{Phosphate solubilization}

Thirteen isolates (a proportion of 0.86) were able to solubilize tri-calcium phosphate on PVK agar medium. Nine of these isolates also were capable of solubilizing phosphate on NBRIY medium. Isolates KBS5-F, KBE9-1 and KBS6-H resulted in the greatest level of phosphate solubilization, resulting in $10 \mathrm{~mm}$ diameter clear zone followed by isolate KBS5$\mathrm{H}$ that gave a clear zone of $7 \mathrm{~mm}$ in diameter (Table 4). Eight other isolates showed some ability to solubilize phosphate on PVK medium resulting in a clear zone ranging between 0.5 and $5 \mathrm{~mm}$ in diameter.
The same isolates, which effectively solubilized phosphate on PVK medium, displayed improved phosphate solubilization ability on NBRIY medium with clear zone diameters of $12 \mathrm{~mm}$ (KBS6-H, KBS9-1) and $14.5 \mathrm{~mm}$ (KBS5-F). Similarly isolates KBS9-R and KBE7-8, both of which tested positive for phosphate solubilization on PVK medium (clear zone of $5 \mathrm{~mm}$ in diameter each) solubilized phosphate better on NBRIY medium (clear zone of $8.5 \mathrm{~mm}$ and $10 \mathrm{~mm}$ diameter, respectively) (Table 4).

\section{Identification of bacterial isolates}

The three isolates obtained from the sorghum rhizosphere in Ethiopia and which resulted in significant growth promotion in sorghum in the present study have been identified as Bacillus cereus. The proportions of species identified as effective isolates from the rhizosphere of grasses and rhizoplane of roots (South African isolates) were 0.30 B. cereus, 0.23 Chryseomonas luteola, $0 \cdot 15$ each of Serratia marcescence and Sphingomonas paucimobilis and 0.08 each of Stenotrophomonas maltophilia and Brevibacterium laterosporus (Table 6). 
Table 5. LS means for shoot fresh weight and dry weight and grouping of isolates according to the multiple t-distribution test Note: Groups 1, 2 and 3 were formed by applying the multiple t-distribution tests of Gupta \& Panchapakesan (1979) at $P \leqslant 0 \cdot 01$

\begin{tabular}{|c|c|c|c|c|c|c|c|c|c|c|c|}
\hline \multicolumn{6}{|c|}{ Isolates from sorghum rhizosphere } & \multicolumn{6}{|c|}{ Isolates from grasses rhizosphere } \\
\hline \multicolumn{3}{|c|}{ Shoot fresh weight $(\mathrm{g})$} & \multicolumn{3}{|c|}{ Shoot dry weight (g) } & \multicolumn{3}{|c|}{ Shoot fresh weight (g) } & \multicolumn{3}{|c|}{ Shoot dry weight (g) } \\
\hline Isolates & $\begin{array}{c}\text { Ranked } \\
\text { mean }\end{array}$ & Group & Isolates & $\begin{array}{c}\text { Ranked } \\
\text { mean }\end{array}$ & Group & Isolates & $\begin{array}{c}\text { Ranked } \\
\text { mean }\end{array}$ & Group & Isolates & $\begin{array}{c}\text { Ranked } \\
\text { mean }\end{array}$ & Group \\
\hline Control & $0 \cdot 7980$ & 1 & Control & $0 \cdot 3063$ & 1 & Control & 0.6230 & 1 & Control & $0 \cdot 0467$ & 1 \\
\hline KBE5-2 & $1 \cdot 0830$ & 1 & KBE6-3 & $0 \cdot 3373$ & 1 & KBS10-E & $1 \cdot 5330$ & 1 & KBS10-E & $0 \cdot 3500$ & 1 \\
\hline KBE8-2 & $1 \cdot 1532$ & 2 & KBE4-3 & 0.4071 & 1 & KBS6-11 & $2 \cdot 8330$ & 1 & KBS6-17 & $0 \cdot 3600$ & 1 \\
\hline KBE8-3 & $1 \cdot 1987$ & 2 & KBE8-3 & $0 \cdot 4144$ & 1 & KFP9-K & $2 \cdot 9333$ & 1 & KBS9-R & $0 \cdot 3800$ & 1 \\
\hline KBE1-7 & $1 \cdot 1789$ & 2 & KBE5-2 & $0 \cdot 4168$ & 1 & KBS6-2 & 2.9670 & 1 & KBS1-T & $0 \cdot 4500$ & 1 \\
\hline KBE4-3 & $1 \cdot 2240$ & 2 & KBE1-7 & $0 \cdot 4346$ & 1 & KBS9-R & $3 \cdot 1000$ & 1 & KBS6-1 & 0.4767 & 1 \\
\hline KBE6-3 & $1 \cdot 2410$ & 2 & NAE4-1 & $0 \cdot 4505$ & 1 & KBS1-T & $3 \cdot 2300$ & 1 & NAS2-B & $0 \cdot 4867$ & 1 \\
\hline NAE9-5 & $1 \cdot 2710$ & 2 & NAE2-8 & $0 \cdot 4618$ & 1 & KBS5-H & $3 \cdot 6770$ & 1 & KBS9-H & 0.4967 & 1 \\
\hline NAE2-8 & $1 \cdot 2970$ & 2 & NAE5-7 & $0 \cdot 4669$ & 1 & KBS6-17 & $3 \cdot 8400$ & 1 & KBS1-J & 0.5067 & 1 \\
\hline NAE4-1 & $1 \cdot 3160$ & 3 & KBE7-6 & 0.4710 & 1 & KBS2-12 & $3 \cdot 9000$ & 1 & KFP9-K & 0.5600 & 2 \\
\hline NAE5-7 & $1 \cdot 3330$ & 3 & KBE8-2 & $0 \cdot 4842$ & 1 & NAS1-6 & $3 \cdot 9000$ & 1 & NAS6-N & 0.5600 & 2 \\
\hline KBE5-7 & $1 \cdot 3810$ & 3 & NAE9-5 & $0 \cdot 5055$ & 1 & KBS1-F & $3 \cdot 9330$ & 1 & KBS5-H & $0 \cdot 5800$ & 2 \\
\hline KBE6-1 & 1.4360 & 3 & KBE6-1 & 0.5171 & 1 & KBS1-J & $4 \cdot 0330$ & 1 & KBS6-11 & $0 \cdot 6000$ & 2 \\
\hline NAE7-1 & $1 \cdot 4430$ & 3 & KBE5-3 & $0 \cdot 5172$ & 1 & NAS2-B & $4 \cdot 0670$ & 1 & KBS1-F & 0.6833 & 2 \\
\hline KBE5-3 & $1 \cdot 4740$ & 3 & KBE5-7 & $0 \cdot 5449$ & 1 & NAS6-N & $4 \cdot 2370$ & 1 & NAS1-6 & $0 \cdot 7233$ & 2 \\
\hline KBE9-1 & $2 \cdot 0040$ & 3 & NAE7-1 & 0.5469 & 1 & KBS9-H & $4 \cdot 2670$ & 1 & KBS2-12 & 0.7433 & 2 \\
\hline KBE9-4 & $2 \cdot 1260$ & 3 & KBE9-1 & 0.7073 & 2 & KFP9-E & $5 \cdot 5000$ & 2 & KFP9-E & 0.9900 & 2 \\
\hline KBE5-8 & $2 \cdot 7150$ & 3 & KBE5-8 & 0.7323 & 2 & KBS5-F & $6 \cdot 8000$ & 2 & KBS5-F & 0.9967 & 2 \\
\hline KBE5-1 & $3 \cdot 5210$ & 3 & KBE9-4 & 0.7788 & 2 & KBS6-H & 6.9670 & 2 & NAS4-3 & $1 \cdot 3633$ & 3 \\
\hline \multirow{2}{*}{ KBE7-8 } & $3 \cdot 8320$ & 3 & KBE5-1 & $1 \cdot 1545$ & 3 & KBS9-B & $7 \cdot 3600$ & 2 & KBS6-H & $1 \cdot 7200$ & 3 \\
\hline & & & KBE7-8 & $1 \cdot 1709$ & 3 & NAS4-3 & $9 \cdot 3670$ & 2 & KBS9-B & 1.7933 & 3 \\
\hline
\end{tabular}

\section{DISCUSSION}

The role of plant growth promoting bacteria in increasing the growth and yield of various crops such as wheat (Khalid et al. 2004), rice (Thakuria et al. 2004), maize (Berge et al. 1991) and many others has been reported in the past. Pacovsky (1990) reported the effect of inoculation of Azosprillum sp. on sorghum growth, indicating that the presence of Azosprillum in the endorhizosphere of sorghum had a positive impact on host growth. Later, Rashad et al. (2002) investigated the growth promoting effects of Rhizobium and Bradyrhizobium inoculation in sorghum. However, other reports on the occurrence of PGPRs in the sorghum rhizosphere, and their effect on the growth of this crop are scarce.

Three isolates obtained from the rhizosphere of sorghum and 16 isolates from the rhizosphere of grasses resulted in significant growth increase in sorghum under greenhouse conditions as measured by the parameters in this study. Isolates KBE7-8, KBE51 and KBE9-1 from sorghum rhizosphere and isolates NAS4-3, KBS5-H, KBS9-H and KFP9-K from the rhizosphere and rhizoplane of grasses, all of which resulted in significant growth increase in one or more parameters were identified as $B$. cereus. The results obtained in the current study concur with several other studies which elucidated the growth promoting activity of isolates of B. cereus. Chen et al. (1994) reported that inoculation with $B$. cereus increased grain yield in rapeseed. In a similar study, Xia et al. (1990) reported that B. cereus strain 83-10 promoted growth and increased grain yield of rapeseed in a repeated field trial. In the current study, the B. cereus isolates significantly promoted early growth of sorghum. The results indicate that the mechanisms involved in the enhancement of growth in sorghum in the current study include the production of the auxin IAA, siderophores and the ability to solubilize phosphate (Kloepper et al. 1989).

All seven strains of B. cereus were efficient in colonizing sorghum roots and significantly increased root length. The stimulation of root growth may be attributed to the production of IAA in culture at a concentration ranging between 4.2 and $22.8 \mu \mathrm{g} / \mathrm{ml}$ in the presence of $2 \mathrm{mg}$ tryptophan/g of nutrient broth solution. Patten \& Glick (2002) reported the production of IAA by wild type Pseudomonas putida from as little as $0.5 \mu \mathrm{g} / \mathrm{ml}$ in the absence of tryptophan to as much as $32.7 \mu \mathrm{g} / \mathrm{ml}$ in the presence of 
Table 6. Identification of the most effective South African and Ethiopian rhizobacterial isolates based on the API system and $16 S$ rDNA sequencing

\begin{tabular}{|c|c|c|c|c|c|c|c|c|}
\hline $\begin{array}{l}\text { Bacterial } \\
\text { isolates }\end{array}$ & $\begin{array}{l}\text { Gram } \\
\text { reaction }\end{array}$ & Endospore* & $\begin{array}{c}\text { Catalase } \\
\text { test }\end{array}$ & $\begin{array}{c}\text { Cytochrome } \\
\text { oxidase }\end{array}$ & $\begin{array}{l}\text { Motility } \\
\text { test }\end{array}$ & $\begin{array}{l}\mathrm{O} / \mathrm{F} \\
\text { reaction } \dagger\end{array}$ & $\begin{array}{l}\text { Bacterial } \\
\text { species }\end{array}$ & $\begin{array}{c}\text { Type of } \\
\text { identification }\end{array}$ \\
\hline KBE7-8 & + & + & + & + & Motile & $\mathrm{Nd}$ & Bacillus cereus & $\begin{array}{l}\text { 16S rDNA } \\
\text { sequencing }\end{array}$ \\
\hline KBE5-1 & + & + & + & + & Motile & $\mathrm{Nd}$ & B. cereus & $\begin{array}{l}\text { 16S rDNA } \\
\text { sequencing }\end{array}$ \\
\hline KBS9-H & + & + & + & - & Not motile & $\mathrm{Nd}$ & B. cereus & $\begin{array}{l}\text { 16S rDNA } \\
\text { sequencing }\end{array}$ \\
\hline NAS4-3 & + & + & + & + & Motile & $\mathrm{Nd}$ & B. cereus & API-50CHB \\
\hline KFP9-K & + & + & + & + & - & $\mathrm{Nd}$ & B. cereus & $\begin{array}{l}\text { 16S rDNA } \\
\text { sequencing }\end{array}$ \\
\hline KBE9-1 & + & + & + & + & Motile & $\mathrm{Nd}$ & B. cereus & $\begin{array}{l}\text { 16S rDNA } \\
\text { sequencing }\end{array}$ \\
\hline KBS5-H & + & - & + & - & Motile & $\mathrm{Nd}$ & B. cereus & $\begin{array}{l}16 \mathrm{~S} \text { rDNA } \\
\text { sequencing }\end{array}$ \\
\hline KBS6-H & - & - & + & + & Motile & Oxidative & $\begin{array}{l}\text { Serratia } \\
\text { marcescens }\end{array}$ & $\begin{array}{l}\text { 16S rDNA } \\
\text { sequencing }\end{array}$ \\
\hline KBS9-R & - & - & + & - & Motile & Oxidative & S. marcescens & $\begin{array}{l}\text { 16S rDNA } \\
\text { sequencing }\end{array}$ \\
\hline KBS5-F & - & - & - & + & Motile & Oxidative & $\begin{array}{l}\text { Chryseomonas } \\
\text { luteola }\end{array}$ & API $20 \mathrm{NE}$ \\
\hline NAS1-6 & - & - & + & + & Motile & Oxidative & C. luteola & API $20 \mathrm{NE}$ \\
\hline KBS6-11 & - & - & + & + & Not motile & Oxidative & C. luteola & API $20 \mathrm{NE}$ \\
\hline NAS2-B & - & - & + & + & Not motile & Oxidative & $\begin{array}{l}\text { Sphingomonas } \\
\text { puacimobilis }\end{array}$ & API $20 \mathrm{NE}$ \\
\hline KBS1-T & - & - & + & + & Not motile & Oxidative & S. puacimobilis & API $20 \mathrm{NE}$ \\
\hline KBS2-12 & + & - & + & - & Not motile & $\mathrm{Nd}$ & $\begin{array}{l}\text { Brevibacterium } \\
\text { laterosporus }\end{array}$ & API $20 \mathrm{NE}$ \\
\hline KBS9-B & - & - & + & - & Motile & Oxidative & $\begin{array}{l}\text { Stenotrophomonas } \\
\text { maltophilia }\end{array}$ & API $20 \mathrm{NE}$ \\
\hline
\end{tabular}

$*+$, endospore present; -, endospore absent.

$\dagger \mathrm{Nd}=$ oxidation fermentation test not conducted.

* Sequencing of the bacterial 16S rDNA was performed for those isolates identified by the API system with less than $0 \cdot 08$ identity with the isolates on the database.

$500 \mu \mathrm{g} / \mathrm{ml}$ tryptophan which resulted in the development of the host plant root system.

Many root-associated bacteria have been reported to produce IAA in culture media (Patten \& Glick 1996, 2002). In the present study, we have observed a positive linear relationship with significant $r$ value $(r=0.57, P<0 \cdot 05)$ between the in vitro IAA production and increase in root length. Asghar et al. (2002) and Khalid et al. (2004) have previously reported a positive correlation between in vitro auxin production and increased growth following application of PGPR. The ability to produce IAA by the most effective isolates in our study might have contributed to the successful root colonization by these isolates (Suzuki et al. 2003) and resulted in significant increase in root growth. In addition to IAA production, the solubilization of phosphate in the rhizosphere is the most common mode of action which may increase nutrient availability to host plants (Vessey 2003). Clear zone formation on Pikovskaya (PVK) medium has been effected by B. cereus strains KBE7-8, KBE9-1, KBS9-H and KBS5-H all of which increased growth in sorghum in the current study.

In the current study, the majority of the bacteria isolated from the rhizosphere of sorghum were Bacillus spp., whereas the rhizosphere of grasses from the Nylsvlei Nature Reserve also was colonized by Gram-negative isolates such as Serratia marcesence, C. luteola, S. maltophilia and S. paucimobilis. Sorghum is an annual crop, while the majority of grasses tested were perennials. Furthermore, the continuous rhizodeposition of carbon from plant roots results in complex chemical and biological interactions in the soil resulting in microbial diversity (Singh et al. 2007). Thus, more growth promoting bacteria were isolated from the grassland soils than from sorghum fields in the current study. 
(a) (i)

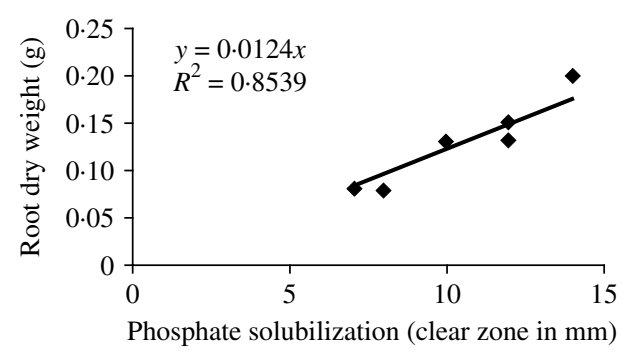

(b) (i)

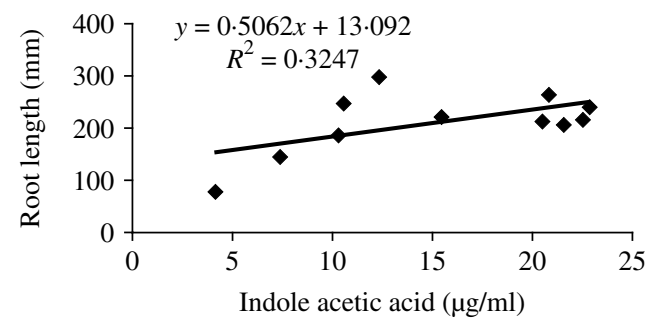

(ii)

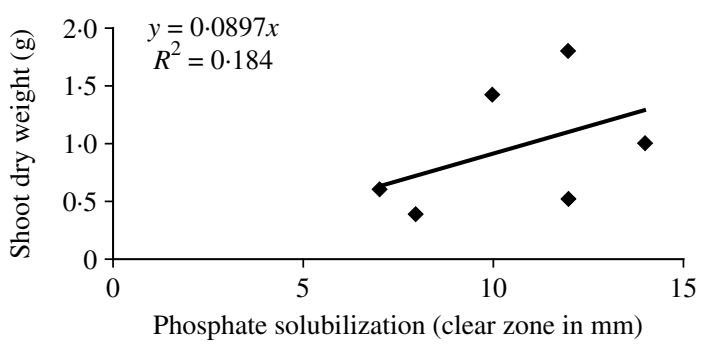

(ii)

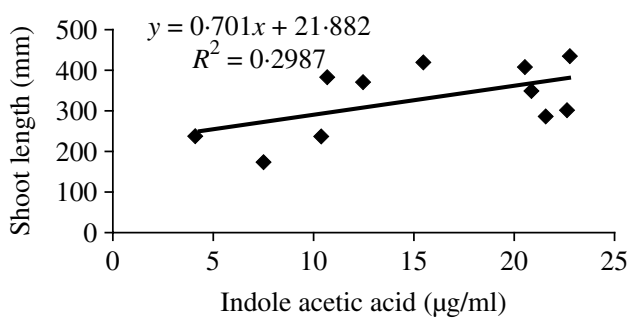

Fig. 3. (a) Relationship between phosphate solubilization and shoot/root dry weight: (i) $r$ is significant $(P=0 \cdot 0005)$ for root dry weight and P-solubilization. (ii) $r$ is not significant $(P=0.05)$ for shoot dry weight and P-solubilization. (b) Relationship between Indole-3-acetic acid concentration and root/shoot length: (i) $r$ is significant $(P=0.05)$ for root length and IAA concentration. (ii) $r$ is significant $(P=0 \cdot 05)$ for shoot growth and IAA concentration.

The Gram-negative isolates exhibited one or more of the properties that are associated with growth promotion. S. maltophilia, Serratia marcescens and $C$. luteola have been reported as members of the naturally occurring rhizosphere community (Lottman et al. 1999; Donnate-Correa et al. 2004) and show plant growth promotion properties and biocontrol activities against plant pathogens (Weller 1998; Whipps 2001). However as several members of these genera exist as potential human pathogens in the rhizosphere of diverse plants (Berge et al. 2005), the necessary toxicological tests would need to be conducted before developing these strains for commercial applications.

Gram-negative isolates that resulted in significant increase in growth of sorghum in this study also showed the ability to solubilize phosphate on PVK medium by producing clear zones ranging from 3 to $10 \mathrm{~mm}$ in diameter. Clear zone formation was greater when $C$. luteola KBS5-F and S. marcescence KBS6-H were inoculated on a modified PVK medium. These results are in agreement with the findings of Nautiyal (1999), who demonstrated that by omitting yeast extract from PVK medium, higher phosphate solubilization was achieved by Pseudomonas sp. For some isolates such as S. maltophilia KBS9-B, phosphate solubilization was similar with and without yeast extract. For other isolates like B. cereus KBS5-H and $S$. paucimobilis KBS1-T, the extent of solubilization (observed from the clear zone formation) decreased or was not observed when yeast extract was removed from the medium. The main effect of phosphate solubilization on plant growth is an increase in biomass and P content (Bashan \& de-Bashan 2004) and in this study a positive linear correlation was obtained between phosphate solubilization and root dry weight $(r=0.92, P<0.005)$ with the most effective bacterial isolates.

S. marcescens strain KBS6-H resulted in growth promotion of sorghum by increasing shoot fresh and dry weights, chlorophyll content as well as root length. Strains of Serratia spp. have previously been reported by other researchers (Zhang et al. 1996; Dashti et al. 1997; Ryu et al. 2005) to promote growth in different crops by a variety of modes of action under controlled conditions. In the current study, S. marcescens (KBS6-H) colonized the roots at a higher level $\left(10^{8} \mathrm{cfu} / \mathrm{g}\right)$ than the control and other non effective isolates and tested positive for the production of siderophores, IAA and phosphate solubilization. Interestingly, the other $S$. marcescens strain (KBS9-R) did not stimulate plant growth although it produced siderophores, IAA and solubilized phosphate. This probably indicates that the ability to manifest these modes of actions by a given bacterium may not necessarily mean that the bacterium is a PGPR (Vessey 2003). Cattelan et al. (1999), for instance, found that out of five rhizosphere isolates which tested positive for $\mathrm{P}$ solubilization, only two had a positive effect on soybean seedling growth. In a 
separate study we conducted, S. marcescens (KBS9$\mathrm{R})$ proved to be very effective in the suppression of Pythium ultimum (Idris et al. 2008) and Fusarium oxysporum (unpublished data) associated with soilborne diseases of sorghum.

Although previous reports on S. maltophilia focussed mainly on their biocontrol activity (Kobayashi et al. 1995, 2002; Zhang et al. 2000), in this study, we isolated one strain (S. maltophilia strain KBS9-B) which resulted in a significant increase in all the seven parameters tested to evaluate growth promotion in sorghum. This strain showed a high root colonization ability $\left(10^{8} \mathrm{cfu} / \mathrm{g}\right)$, phosphate solubilization and production of IAA. Sturz et al. (2001) recovered plant growth promoting strains of $S$. maltophilia from the root zones of quack grass (Agropyron repens (L.) Beauv) which significantly increased the biomass of shoots and roots in in vitro bacterization studies. Our result also concurs with that of Donnate-Correa et al. (2004) who previously isolated IAA producing strains of $S$. maltophilia and C. luteola from the rhizosphere of the perennial legume tagasate. C. luteola KBS5-F did not however produce IAA in this study. Their growth promoting ability in the current study may therefore be associated with production of siderophores and solubilization of phosphate (Glick 1995; Rodriguez \& Fraga 1999).

Enhanced iron nutrition resulting in increased plant growth can also be achieved due to the ability of some plants to bind and release iron from bacterial iron-siderophore complexes and utilizing the iron for growth (Bashan \& de-Bashan 2004). Of the isolates that resulted in significant growth promotion in the current study, $S$. marcescens KBS6-H, C. luteola KBS5-F and B. cereus KBE9-1 produced siderophores with characteristic yellow halo formation on CAS agar plates. The results suggest that the bacterial isolates might have increased sorghum growth by the action of siderophores which is known to sequester iron from the soil and provide it to the plant (Katiyar \& Goel 2004).

The main conclusions from the current study are that rhizobacteria isolated from the rhizosphere and rhizoplane of grasses in South Africa and from the rhizosphere of sorghum in Ethiopia have the ability to enhance growth of sorghum under greenhouse conditions. It is also concluded that perennial grasses harbour more growth promoting bacteria in their rhizosphere than sorghum occurring in commercial fields. The present study also provides information on the application of rhizobacteria as inoculants in agriculture in Ethiopia and South Africa. However, in order to develop the best performing PGPR strains for commercial applications, further selection and screening in field trials is being conducted. Additional studies on modes of action need to be conducted to elucidate the role of the bacterial isolates in increasing phosphorous and iron uptake by the plants.

\section{REFERENCES}

Asghar, H. N., Zahir, Z. A., Arshad, M. \& Khaliq, A. (2002). Relationship between in-vitro production of auxin by rhizobacteria and their growth promoting activities in Brassica juncea L. Biology and Fertility of Soils 35, 231-237.

Asghar, H. N., Zahir, Z. A. \& Arshad, M. (2004). Screening rhizobacteria for improving the growth, yield, and oil content of canola (Brassica napus L.). Australian Journal of Agricultural Research 55, 187-194.

Bashan, Y. \& De-Bashan, L. E. (2004). Plant growth promoting bacteria. In Encyclopaedia of Soils in the Environment, vol. 1 (Ed. D. Hillel), pp. 103-115. Oxford, UK: Elsevier.

Berge, G., Eberl, L. \& Hartman, A. (2005). The rhizosphere as areservior for opportunistic human pathogenic bacteria. Environmental Microbiology 7, 1673-1685.

Berge, O., Heulin, T. \& Balandreau, J. (1991). Diversity of diazotroph populations in the rhizosphere of maize (Zea mays L.) growing on different French soils. Biology and Fertility of Soils 11, 210-215.

Bertrand, H., Nalin, R., Bally, R. \& Cleyet-Marel, J. C. (2001). Isolation and identification of the most efficient plant growth promoting bacteria associated with canola (Brassica napus). Biology and Fertility of Soils 33, 152156.

Bloemberg, G. V. \& Lugtenberg, B. J. J. (2001). Molecular basis of plant growth promotion and biocontrol by rhizobacteria. Current Opinion in Plant Biology 4, 343-350.
Cattelan, A. J., Hartel, P. G. \& Fuhrmann, J. J. (1999). Screening for plant growth-promoting rhizobacteria to promote early soybean growth. Soil Science Society of America Journal 63, 1670-1680.

Chen, Y., Mei, R., Lu, S., Liu, L. \& Kloepper, J. W. (1994). The use of yield increasing bacteria as plant growth promoting rhizobacteria in Chinese agriculture. In Management of Soil Borne Diseases (Eds U. K. Gupta \& R. Utkhede), pp. 165-184. New Delhi: M/S Narosa Publishing House.

Coenye, T., Falsen, E., Vancanneyt, M., Hoste, B., Govan, J. R. W., Kersters, K. \& Vandamme, P. (1999). Classification of Alcaligens faecalis like isolates from the environment and human clinical samples as Ralstonia giladii sp. nov. International Journal of Systematic Bacteriology 49, 405-413.

Cоок, R. J. (2002). Advances in plant health management in the 20th century. Annual Review of Phytopathology 38, 95-116.

Dashti, N., Zhang, F., Hynes, R. \& Smith, D. L. (1997). Application of plant growth-promoting rhizobacteria to soybean (Glycine max (L.) Merr.) increases protein and dry matter yield under short-season conditions. Plant and Soil 188, 33-41.

DAvis, M. A. \& Bockus, W. W. (2001). Evidence for a Pythium species as a chronic yield reducer in a continuous grain sorghum field. Plant Disease 85, 780784 . 
Donnate-Correa, J., Leon-Barrios, M. \& PerezGaldonA, R. (2004). Screening for plant growth promoting rhizobacteria in Chamayecytisus proliferus (tagasaste), a forage tree shrub legume endemic to the Canary islands. Plant and Soil 266, 261-272.

FAO (1999). FAO Quarterly Bulletin of Statistics, vol. 12. Rome, Italy: FAO.

FAOSTAT (2005). Statistics Database. Available online at: http://faostat.fao.org (verified 13 October 2008).

Glick, B. R. (1995). The enhancement of plant growth by free living bacteria. Canadian Journal of Microbiology 41, $109-117$.

Gregersen, T. (1978). Rapid method for distinction of Gram-positive bacteria. European Journal of Applied Microbiology and Biotechnology 5, 123-127.

Gupta, R., Singal, R., Sankar, A., Chander, R. M. \& Kumar, R. S. (1994). A modified plate assay for screening phosphate solubilizing microorganisms. Journal of General and Applied Microbiology 40, 255-260.

Gupta, S. S. \& Panchapakesan, S. (1979). Multiple Decision Procedures: Theory and Methodology of Selecting and Ranking Populations. New York: John Wiley \& Sons.

Han, D. Y., Coplin, D. L., Bauer, W. D. \& Hoitink, H. A. J. (2000). A rapid bioassay for screening rhizosphere microorganisms for their ability to induce systemic resistance. Phytopathology 90, 327-332.

House, L. R. (1995). Sorghum: one of the world's great cereals. African Crop Science Journal 3, 135-142.

Hugh, R. \& Leifson, H. (1953). The taxonomic significance of fermentative versus oxidative Gram-negative bacteria. Journal of Bacteriology 66, 24-26.

Huluka, M. \& Esele, J. P. E. (1992). Sorghum diseases in Eastern Africa. In Sorghum and Millet Diseases: a Second World Review (Eds W. J. A. De-Millano, R. A. Frederiksen, L. K. Mughogo \& D. J. Bengston), pp. 36-39. Pathacheru, India: ICRISTAT.

Igual, J. M., Valverde, A., Cervantes, E. \& Velazquez, E. (2001). Phosphate solubilizing bacteria as inoculants for agriculture: use of updated molecular techniques in their study. Agronomie 21, 561-568.

Idris, H. A., Labuschagne, N. \& Korsten, L. (2008). Suppression of Pythium ultimum root rot of sorghum by rhizobacterial isolates from Ethiopia and South Africa. Biological Control 45, 72-84.

Katiyar, V. \& Goel, R. (2004). Siderophore mediated plant growth promotion at low temperature by mutant of fluorescent pseudomonad. Plant Growth Regulation 42, $239-244$

Khalid, A., Arshad, M., Zahir, Z. A. \& Khaliq, A. (1997). Potential of plant growth promoting rhizobacteria for enhancing wheat (Triticum aestivum L.) yield. Journal of Animal and Plant Sciences 7, 53-56.

Khalid, A., Arshad, M. \& Zahir, Z. A. (2004). Screening plant growth promoting rhizobacteria for improving growth and yield of wheat. Journal of Applied Microbiology 96, 473-480.

King, E. O., Ward, M. K. \& Ranney, D. E. (1954). Two simple media for the demonstration of pyocyanin and fluorescin. Journal of Laboratory and Clinical Medicine 44, 301-307.

Kloepper, J. W., Scher, F. M., Laliberté, M. \& Tipping, B. (1986). Emergence promoting rhizobacteria: description and implications for agriculture. In Iron, Siderophores and
Plant Disease (Ed. T. R. Swinburne), pp. 155-164. Boca Raton, FL: CRC Press.

Kloepper, J. W., Lifshitz, R. \& Zablotowicz, R. M. (1989). Free living soil bacterial inocula for enhancing crop productivity. Trends in Biotechnology 7, 39-43.

Kobayashi, D. Y., Guglielmoni, M. \& Clarke, B. B. (1995). Isolation of the chitinolytic bacteria Xanthomonas maltophilia and Serratia marcescens as biological control agents for summer patch disease of turfgrass. Soil Biology and Biochemistry 27, 1479-1487.

Kobayashi, D. Y., Reedy, R. M., Bick, J. A. \& Oudemans, P. V. (2002). Characterization of chitinase gene from Stenotrophomonas maltophilia strain $34 \mathrm{~S} 1$ and its involvement in biological control. Applied and Environmental Microbiology 68, 1047-1054.

Kovacs, N. (1956). Identification of Pseudomonas pyocyanae by the oxidase reaction. Nature 178, 703 .

Lottman, J., Heuer, H., Smalla, K. \& Berg, G. (1999). Influence of transgenic T4-lysozyme-producing potato plants on potentially beneficial plant associated bacteria. FEMS Microbiology Ecology 29, 365-367.

Mayak, S., Tirosh, T. \& Glick, B. R. (2001). Stimulation of the growth of tomato, pepper and mung bean plants by the plant growth promoting bacterium Enterobacter cloacae CAL3. Biological Agriculture and Horticulture 19, 261-274.

Mohran, Z. S., Arthur, R. R., Oyufo, B. A., Perusky, L. F., Wasfy, M. O., Ismael, T. F. \& Murphy, J. R. (1998). Differentiation of Campylobacter isolates on the basis of sensitivity to boiling in water as measured by PCR-detectable DNA. Applied and Environmental Microbiology 64, 363-365.

Nautiyal, C.S. (1999). An efficient microbiological growth medium for screening phosphate solubilizing microorganisms. FEMS Microbiology Letters 170, 265270.

Nelson, L. M. (2004). Plant growth promoting rhizobacteria (PGPR): prospects for new inoculants. Online Crop Management doi:10.1094/CM-2004-0310-05-RV. Available online at: http://www.plantmanagement network.org/pub/cm/review/2004/rhizobacteria/ (verified 13 October 2008)

Pacovsky, R. S. (1990). Development and growth effects in the Sorghum-Azosprillum association. Journal of Applied Microbiology 68, 555-563.

Patten, C. L. \& Glick, B. R. (1996). Bacterial biosynthesis of indole-3-acetic acid. Canadian Journal of Microbiology 42, 207-220.

Patten, C. L. \& Glick, B. R. (2002). Role of Pseudomonas putida indoleacetic acid in development of the host plant root system. Applied and Environmental Microbiology 68, 3795-3801.

Pikovskaya, R. I. (1948). Mobilization of phosphorus in connection with the vital activity of some microbial species. Microbiologia 17, 362-370.

Rashad, M. H., Ragab, A. A. \& Salem, S. M. (2002). The influence of some Bradyrhizobium and Rhizobium strains as plant growth promoting rhizobacteria on the growth and yield of sorghum (Sorghum bicolor L.) plants. In Plant Nutrition (Eds W. J. Horst, M. K. Schenk, A. Burkert, N. Claassen, H. Flessa, W. B. Frommer, H. Goldbach, H. W. Olfs, V. Romheld, B. Sattelmacher, U. Schmidhalter, S. Schubert, N. V. Wiren \& L. Wittenmayer), pp. 664-665. The Netherlands: Springer. 
Rodriguez, H. \& Fraga, R. (1999). Phosphate solubilizing bacteria and their role in plant growth promotion. Biotechnology Advances 17, 319-339.

Ryu, C.-M., Hu, C.-H., Locy, R. D. \& Kloepper, J. W. (2005). Study of mechanisms for plant growth promotion elicited by rhizobacteria in Arabidopsis thaliana. Plant and Soil 268, 285-292.

Saubidet, M. I., Fatta, N. \& Barneix, A. J. (2002). The effect of inoculation with Azosprillum brasilense on growth and nitrogen utilization by wheat plants. Plant and Soil 245, 215-222.

Schwyn, B. \& Neilands, J. B. (1987). Universal chemical assay for the detection and determination of siderophores. Analytical Biochemistry 160, 47-56.

Singh, B. K., Munro, S., Potts, J. M. \& Millard, P. (2007). Influence of grass species and soil type on rhizosphere microbial community structure in grassland soils. Applied Soil Ecology 36, 147-155.

Sturz, A. V., Matheson, B. G., Arsenault, W., Kimpniski, J. \& Christie, B. R. (2001). Weeds as a source of plant growth promoting rhizobacteria in agricultural soils. Canadian Journal of Microbiology 47, 10131024.

Suzuki, S., He, Y. \& Oyaizu, H. (2003). Indole-3-acetic acid production in Pseudomonas fluorescens HP72 and its association with suppression of creeping bentgrass brown patch. Current Microbiology 47, 138-143.

Thakuria, D., Talukdar, N. C., Goswami, C., Hazarika, S., Boro, R. C. \& KhAN, M. R. (2004). Characterization and screening of bacteria from rhizosphere of rice grown in acidic soils of Assam. Current Science 86, 978-985.

The Guide To Genstat ${ }^{\circledR}$ Release 8 (2005). (Ed. R. W. Payne). Published by VSN International, UK, ISBN 1904375-16-2.

Vessey, J. K. (2003). Plant growth promoting rhizobacteria as biofertilizers. Plant and Soil 255, 571-586.

Weller, D. M. (1998). Biological control of soilborne plant pathogens in the rhizosphere with bacteria. Annual Review of Phytopathology 26, 379-407.

Whipps, J. M. (2001). Microbial interactions and biocontrol in the rhizosphere. Journal of Experimental Botany 52, 487-511.

XiA, L., Ding, X., Li, J. \& MeI, R. (1990). Mechanism of PGPR: influence of PGPR on physiology, resistance, quality and yield of rapeseed. Agriculture Science in Hunan 106, 24-26.

Zahir, Z. A., Akram, M., Arshad, M. \& Khalid, A. (1998). Improving maize yield by inoculation with plant growth promoting rhizobacteria. Pakistan Journal of Soil Science 15, 7-11.

Zhang, F., Dashti, N., Hynes, H. \& Smith, D. L. (1996). Plant growth promoting rhizobacteria and soybean (Glycine max L. Merr.) nodulation and nitrogen fixation at suboptimal root zone temperatures. Annals of Botany 77, 453-459.

Zhang, Z., Yuen, G. Y., Sarath, G. \& Penheiter, A. R. (2000). Chitinase from the plant disease biocontrol agent, Stenotrophomonas maltophilia C3. Phytopathology 91, 204-211. 